\title{
From shared stimuli to preestablished harmony: the development of Quine's thinking on intersubjectivity and objective validity
}

\author{
Gubelmann, Reto
}

\begin{abstract}
W. V. O. Quine is generally seen as one of the foremost empiricists of the twentieth century. For large parts of his career, the label "empiricist" is accurate; in his mature work, however, he integrated decidedly antiempiricist elements in his epistemology. From The Roots of Reference onward, he enlists natural selection and innate cognitive structures to ensure that scientific concepts have a "degree of objective validity." From From Stimulus to Science onward, he also explains the very possibility of communication via a preestablished harmony of innate cognitive structures that is guaranteed by natural selection. This article reconstrues the reasons that compelled Quine to these commitments, and it details the development of Quine's thinking on these topics across more than 3 decades; in particular, the article argues that recognizing that so-called stimulus meanings are private decisively shaped Quine's views. By means of a critical evaluation, the article argues that natural selection can make plausible that scientific concepts have a degree of objective validity - if this Quinean claim is properly understood; in contrast, the article suggests, with recourse to research by Robert C. Richardson, that it is doubtful whether natural selection can underpin the preestablished harmony that Quine requires to explain communication.
\end{abstract}

DOI: https://doi.org/10.1086/703253

Posted at the Zurich Open Repository and Archive, University of Zurich ZORA URL: https://doi.org/10.5167/uzh-174287

Journal Article

Accepted Version

Originally published at:

Gubelmann, Reto (2019). From shared stimuli to preestablished harmony: the development of Quine's thinking on intersubjectivity and objective validity. HOPOS, 9(2):343-370.

DOI: https://doi.org/10.1086/703253 


\section{From Shared Stimuli to Preestablished Harmony: The Development of Quine's Thinking on Intersubjectivity and Objective Validity}

This is a post-peer-review, pre-copyedit version of an article published in HOPOS. The final authenticated version is available online at: at https://doi.org/10.1086/703253

Abstract

Quine is generally seen as one of the foremost empiricists of the $20^{\text {th }}$ century. For large parts of his career, the label "empiricist" is accurate; in his mature work, however, he integrated decidedly anti-empiricist elements in his epistemology. From The Roots of Reference onwards, he enlists natural selection and innate cognitive structures to ensure that scientific concepts have "a degree of objective validity". From From Stimulus to Science onwards, he also explains the very possibility of communication via a preestablished harmony of innate cognitive structures that is guaranteed by natural selection. The article reconstrues the reasons that compelled Quine to these commitments, and it details the development of Quine's thinking on these topics across more than three decades; in particular, the article argues that recognizing that so-called stimulus meanings are private has decisively shaped Quine's views. By means of a critical evaluation, the article argues that natural selection can make plausible that scientific concepts have a degree of objective validity - if this Quinean claim is properly understood; in contrast, the article suggests, with recourse to research by

Robert C. Richardson, that it is doubtful whether natural selection can underpin the preestablished harmony that Quine requires to explain communication. 


\section{Introduction}

W.V. Quine is widely held to have been an empiricist. Often, he himself explicitly adopted this label. For his research until the late 1980s, this categorization is accurate, and it agrees with the readings of Quine's foremost interpreters of these days, such as Gibson (1988). However, as I will argue in this article, in his mature philosophy, he decisively departed from core empiricist convictions. Analogously, Quine has defended a version of physicalism for some of his most productive and influential research periods, whereas his mature philosophy is not recognizably physicalistic anymore. ${ }^{1}$

This article details Quine's development away from this physicalistic empiricism, as it is outlined most forcefully in Word and Object, to the mature account in From Stimulus to Science, which is characterized by heavy use of evolutionary notions and innate cognitive structures. I point out that there were two main reasons that prompted this Quinean development, namely a basic problem in his account of linguistic meaning as well as an unanswered question regarding his philosophy of science: It became clear to Quine that the account given in Word and Object implies that competent speakers of a language are necessarily talking past each other - even on the level of so-called holophrastic observation sentences (compare Burge 2010, 224). ${ }^{2}$ Furthermore, Quine realized that what he called innate quality spaces in Word and Object determine the epistemic success or failure of science, which prompted the question why these quality spaces could provide the ground for what he saw as the undeniable epistemic success of science. The article concludes with a critical evaluation of this double use of natural selection to address both issues. 
The article is structured as follows. In the remainder of this introductory section, I first situate the topic of this article in the broader context of $20^{\text {th }}$ century philosophy of science. Then, I delineate three central concepts for Quine's epistemology and for his theory of meaning. In doing so, I focus on Word and Object, but I also take later writings into account where appropriate. Next, in sections 2 and 3, I detail the problems that Quine recognized with this account from Word and Object, and I delineate the central steps that Quine took to solve these problems. finally, in section 4, I critically assess these solutions.

Quine's struggle with the intersubjectivity of observation sentences belongs to a central debate in the philosophy of science of his century. As a consequence of several seminal publications after WWII, among them Thomas Kuhn's The Structure of Scientific Revolutions (Kuhn, 1962/1996), but also Quine's "Two Dogmas of Empiricism" (Quine, 1951/1980), philosophers of science worried that all observation and empirical evidence in science might be theory-laden, with the consequence that there would be no neutral arbiter between competing models or theories in a scientific dispute: Each side just conceives the world through their respective theory-laden lens, and there cannot be any common epistemic ground between them. As I will show, Quine intended his conception of holophrastic observation sentences to address this worry.

The first of the three central Quinean concepts to be introduced is observation sentence. According to Quine, the infant's first steps into language are not the acquisition of words, but rather of observation sentences. In Word and Object, Quine (1960, 35-46) introduces observation sentences as a variant of occasion sentences. The latter "command assent or dissent only if queried after an appropriate prompting stimulation", (Quine 1960, 35-36). In this, they differ from standing sentences such as 'Physical bodies attract each other'. Occasion sentences are easier to learn, as a competent speaker's assent to them depends - to a 
considerable degree - on the respective situation.

Furthermore, in most cases, ${ }^{3}$ an observation sentence has two substantially different modes of existence, or more precisely, of use. First, it can be used as a bona fide structured sentence. In this mode, observation sentences have a grammatical and logical structure and they carry ontological commitments - they are part of a theory of the world. Second, in addition to this conceptually structured and thus theory-laden use, observation sentences have a holophrastic, non-conceptual use (Quine 1992, 8). In this mode, which is the epistemically significant one, observation sentences are meaningful only as a whole, as if they were composed of a single word. ${ }^{4}$ Hence, they do not have any (proto-)logical structure, and they are not committed to any ontology or scientific theory; in other words, they provide theory-neutral checkpoints for scientific inquiry. In the following, if not specified otherwise, with 'observation sentence', I always mean their holophrastic use. For more details on this duality of observation sentences, compare Hylton (2007, 140-48).

To properly appreciate what sets observation sentences apart from other occasion sentences, it is necessary to introduce Quine's concept of stimulus meaning. Often, the meaning of a word is conceived as something that is not located in spacetime. For example, the meaning of the word 'horse' could be said to denote an abstract property which is common to all horses. Quine never accepted such a non-physicalistic conception of meaning. Stimulus meaning is what Quine proposes as a physicalistic reconception of the traditional notion of meaning, as a replacement for it.

Quine $(1960,32)$ defines the stimulus meaning of a sentence $P$ relative to the subject $S$ in the following way. The affirmative stimulus meaning of $P$ for $S$ is the class of all the sensory stimulations - across all the subject's senses - which prompt $S$ to assent to P. Mutatis mutandis, Quine defines the negative stimulus meaning of $P$ for $S$ : it is given by the class of 
stimulations that cause $S$ to reject $P$, and the stimulus meaning of $P$ emerges as the ordered pair of the two classes. ${ }^{5}$ To illustrate this notion of stimulus meaning, consider the sentence "Rabbit!". What is the stimulus meaning of this sentence for our speaker Rudolph? According to the definition above, it consists of the class of the stimulations which would cause Rudolph to affirm the sentence together with the class of stimulations which would cause him to deny it. $^{6}$

Quine $(1960,44-45)$ introduces the notion of observationality to set the stimulus meaning of observation sentences apart from the stimulus meaning of other sentences. A sentence is low on observationality if the intersubjective variability of its stimulus meaning is high. Consider the following two examples. On the one hand, the observationality of "A bachelor!" is supposedly very low, as your inclination to assent to it simply depends on whether you know the person in question or not - and not on some observable feature of the respective scene (putting aside things like wedding rings, or their absence). Thus, the stimulus meaning of "A bachelor!" differs among people according to their different social relationships. On the other hand, the observationality of "Red!" is very high, since the stimulus meaning of it is quite uniform among competent English speakers: according to Word and Object, we are all prepared to agree to this latter sentence if, and only if, our retina is stimulated at a certain wavelength. Generally speaking, the stimulus meaning of a given observation sentence is intersubjectively (almost) invariant.

Since observation sentences, taken holophrastically, are directly associated with an intersubjectively invariant stimulus meaning, to judge whether a given observation sentence is true in a given situation does not presuppose any linguistic knowledge. Therefore, the infant has something that she shares with her parent before she can rely on a shared language, and which ipso facto can serve as the gateway to language. Furthermore, thanks to the 
intersubjective invariability and theory-freedom of the stimulus meanings of observation sentences, even proponents of radically different theories will affirm these observation sentences in the same situations, that is, when stimulated similarly in the relevant respect. Thus, holophrastic observation sentences can provide theory-neutral checkpoints for scientific theorizing.

However, even if we grant that observation sentences, used holophrastically, do not presuppose linguistic knowledge, or a theory of the world, this does not adequately explain how they can function as the gateway to language. Regarding the sheer multiplicity of causal patterns of sensory stimulations which accompany any of the parent's utterings of "Red!" sounds, smells, visual patterns, etc. - it is highly unlikely that the child is able to identify the important aspect of this torrential input, namely the presence of a red surface, of this torrential input. Quine addresses this problem with his concept of innate quality spaces (which he later calls 'innate senses of similarity' and finally 'innate similarity standards'). To acquire her first observation sentences, Quine holds, the child must be inclined to give more weight to the relevant similarities than to the irrelevant ones - where the relevant similarities are those which matter for the meanings of the observation sentences that the infant tries to learn. ${ }^{7}$

According to Quine (1960, 83), the child is biased to generalize along the aspects of the stimuli which matter for her parent, namely the red patch in the visual field, as opposed to, say, a gust of wind. The reason for this bias is that the infant's quality spaces situate global stimuli involving red close together, instead of grouping all those global stimuli where a certain distinctive gust of wind is present. This way, the child is able to inductively generalize the stimulus meanings of her first observation sentences. If we remember that the stimulus meanings of observation sentences are uniform among the community of competent speakers, 
it follows that, by having thus learned these observation sentences, the child has learned the meaning of her first words ${ }^{8}$ - a meaning, notably, that she shares with her parent.

Using the behaviorist vocabulary that Quine held in high esteem at the time of Word and Object, this yields a rather neat and physicalistic picture of how the infant makes her first steps into language: having uttered at random something along the lines of "Red!" in the presence of a red surface, the parent rewards the infant. As the infant likes being rewarded, she repeats the same sound on occasions when it is stimulated in a similar way, which means that it has started using the observation sentence "Red!" correctly. Since the possible respects in which another stimulation could be similar to the relevant one are legion, the child needs a quality space that is both biased towards the relevant similarities and similar to the one of her parent. Thanks to this quality space, the infant can associate the correct stimulus meaning with "Red!", a stimulus meaning which is intersubjectively (almost) invariant. In the same way, Quine ensures that two scientists proposing different theories do share a minimal common ground to decide which theory is empirically superior: their theoretical differences notwithstanding, both associate the same stimulus meaning with the same holophrastic observation sentences.

\section{Two Challenges for This Quinean Account}

The first challenge for this Quinean account of how language connects us to the world and to our fellow human beings stems from the function that the quality spaces play in this account. If these quality spaces determine the fundamental touchstones for scientific theorizing, then the epistemic success of scientific theorizing is closely tied to the epistemic standing of these quality spaces. In other words: unless these quality spaces are such that they give us a good basis for scientific theorizing, it is very hard to see how the scientific enterprise could ever 
bear any epistemic fruit, given the fundamental role played by these quality spaces. Quine nowhere mentions this epistemic significance of quality spaces in Word and Object, but he discusses it in Natural Kinds as well as in The Roots of Reference - with strongly differing conclusions. Quine $(1974,19)$ came to conceive this issue as the question regarding the objective validity of scientific theories.

The second problem with this picture is, to put it simply, that stimulus meanings are private (in the sense that they cannot be shared by different speakers). The first article in which Quine explicitly acknowledges the fact that global stimuli are private - and recognizes its relevance for his theory of meaning and evidence - is Propositional Objects. Quine (1969a, viii) writes that he held Propositional Objects as a lecture in 1965 . Thus, the following remarkable passage probably dates from no more than five years after the publication of Word and Object:

It seems vital that in correlating one subject's verbal behavior with another's, for instance as a basis for translating one language into another, we be able to equate one subject's stimulation to another's. Yet how are we to do so? If we construe stimulation patterns my way, we cannot equate them without supposing homology of receptors; and this is absurd, not only because full homology is implausible, but because it surely ought not to matter. (Quine 1969c, 157)

Here, Quine explicitly acknowledges that his notion of stimulus meaning and thus of communication in general depends on homology of sensory receptors, which is implausible. ${ }^{9}$ Why is this the case?

Homology of sensory receptors, which is a condition for the possibility of intersubjectively shared stimulus meaning, is not required when the focus is restricted to one single subject. 
When asked for a verdict on an observation sentence in two different situations, a competent speaker behaves similarly when stimulated in a similar way: she assents to it when confronted with a stimulation which falls in the range of the sentence's affirmative stimulus meaning, and she dissents when the stimulation belongs to the sentence's negative stimulus meaning. However, pace the claim of Word and Object, these stimulus meanings are private. This means that two witnesses of the same scenario who agree on the truth of an observation sentence cannot be said to witness the same situation sensu stricto, that is, they cannot be said to be stimulated alike. In Propositional Objects, Quine first states that, in the context of firstlanguage acquisition, sensory surfaces are the best "boundaries" to define input, but that "when we come to the seemingly essential business of saying what it is for two people to be stimulated alike, we tangle with the myth of homologous nerve endings" (Quine 1969c, 158). Quine here wrestles with the following issue: if, as a consequence of non-homologous nerve endings, the parent's global stimulus differs from the child's in any given situation (even if the child could take the very same physical position as her parent), then it is misguided to explain the success of the child's inductions by means of a quality space which situates global stimuli. No matter how biased her quality space, the child can never hope to infer a stimulus meaning which is substantially like the parent's because no two speakers associate even approximately similar stimuli with a given observation sentence. In other words, even if there was a remarkable similarity between the quality spaces of the parent and the infant, it would still be useless because there are no similar global stimuli to situate in these spaces (compare Quine 1969c, 158). Hence, the privacy of stimulus meanings undermines the intersubjectivity of Quine's conception of linguistic meanings - even on the level of holophrastic observation sentences.

A simple way to bypass this problem is unavailable to Quine. One could simply accept that 
global proximal stimuli are private but assume the distal stimuli (the intersubjectively invariant objects that cause the proximal stimulations) to be relevant for the purposes of naturalized epistemology. Quine $(1992,41)$ rejects this solution because he is "interested in the flow of evidence from the triggering of the senses to the pronouncements of science". Assuming that infants can refer to objects, if only perceptually, before they even learn their first observation sentence would omit the part of naturalized epistemology in which Quine is highly interested: how did humans develop this ability to refer to objects? It therefore runs counter to the very goal of Quine's project to assume the distal stimulus as basic for firstlanguage acquisition.

In effect, at the end of Propositional Objects, Quine admits that he has no solution to this problem. Regarding the homology problem, he remarks: "I leave you, therefore, with a problem of theoretical formulation that carries no practical problem with it" (Quine 1969c, 159-60). However, as I hope to show in the following, Quine was far from simply leaving this problem to his readers. On the very contrary: he grappled with it for the rest of his publishing career.

\section{Quine's Struggle to Explain Objectivity and Intersubjectivity}

In this section, I trace Quine's attempts, extending over more than three decades, to solve a problem that he recognized around 1965: his conception of intersubjectively (almost) invariant stimulus meaning is based on a factually wrong presupposition, namely on homology of nerve endings. Furthermore, I delineate the evolution of Quine's answer to another question: Why is our science so predictively successful? Since science is epistemically based on observation sentences, and since these observation sentences are in turn shaped by our innate quality space, an answer to this question must involve attributing 
some sort of epistemic validity to these quality spaces.

In the following sections, I show how Quine tried to come to terms with these issues in The Roots of Reference (section 3.2) and From Stimulus to Science (section 3.3). This will show that Quine's final proposal to address the two issues is neither recognizably physicalistic, nor empiricist anymore. Before doing that, I discuss Quine's rather surprising position in Natural Kinds (section 3.1).

\subsection{Natural Kinds: Natural Selection Endows Humans Doubly}

Here is how Quine conceives the central function of quality spaces and their inhabitants in Natural Kinds: “The uniformity of people's quality spaces virtually assures that similar presentations will elicit similar verdicts [emphasis RG]" (Quine 1969b, 125). When compared to Word and Object, this passage conceives of the function of quality spaces in the same way: to guarantee that different humans assent to observation sentences in roughly the same situations. However, in contrast to Word and Object, the inhabitants of quality spaces are now vaguely referred to as 'presentations'.

In light of the privacy of stimulus meanings, it is clear why Quine uses 'presentations' instead of 'stimulations': for the reasons presented above (section 2), a quality space that allocates inevitably different stimulations and hence stimulus meanings cannot ensure intersubjectivity of meaning, no matter how uniform the space itself is. If global stimuli are necessarily different from speaker to speaker, then stimulus meanings, being a part of global stimuli, are necessarily different too, no matter how uniform the respective similarity spaces are. As it were, if you take an apple and an orange and put them into two identical storage systems, the two fruits are still not identical, not shared across the two storage systems. This impasse 
notwithstanding, there are still passages in Natural Kinds where Quine (1969b, 123) refers to an "innate spacing of stimulations" which is at work in first-language acquisition. I submit that this ambiguity in use is indicative of a transitory phase in Quine's theory.

Furthermore, in "Natural Kinds" Quine judges the epistemic validity of innate quality spaces very negatively. This innate sense of similarity is based on colors and thus proves very useful "at the food-gathering level" (Quine 1969b, 127). It is also this innate sense of similarity which is vital for first-language acquisition. Unfortunately, "color is king in our innate quality space, but undistinguished in the cosmic circles" (Quine 1969b, 127). In other words, our innate sense of similarity is useful to survive, but not of much use in understanding the laws behind the experiences; it is a bad guide to the "functionally relevant groupings in nature" (Quine 1969b, 126). This, however, is not the end of the story, as natural selection has been particularly generous towards human beings:

Evidently natural selection has dealt with the conflict by endowing man doubly: with both a color slanted quality space and the ingenuity to rise above it. (Quine 1969b, 128)

Here, Quine proposes the following model to explain the capacities of human beings to both succeed on the "food-gathering level" (Quine 1969b, 127), that is, in their daily affairs, and in theoretical science. When it comes to the former, we are well-off with our inborn quality space. When it comes to exploring the "workings of the cosmic machine revealed by science" (Quine 1969b, 133), however, we are able to transcend this inborn quality space and adopt a spacing of qualities which is fit for theoretical science. ${ }^{10}$

These are Quine's first attempts to address a rather straightforward question suggested by his notion of empirical justification. The quality spaces determine the meaning of observation 
sentences, which in turn serve as checkpoints for scientific theorizing. Hence, the question arises: are these innate quality spaces good guidelines for scientific theorizing? The answer that Quine gives in Natural Kinds is: no, they are not. Therefore, he proposes that scientists must be able to transcend this irrational spacing to properly engage in science. Put differently, according to the answer given in Natural Kinds, the success of science is made possible by our ability to rid ourselves of the animal sense of similarity and to develop the cognitive structures needed to pursue theoretical science; in Quine's words: to move on from "unreason into science" (Quine 1969b, 138).

To sum up, in "Natural Kinds", Quine (1969b) substantially revises the notion of quality space, or sense of similarity, when compared to Word and Object. He introduces the notion of theoretical similarity to explain why our science is successful despite the "brute irrationality of our [innate, RG] sense of similarity" (Quine 1969b, 125). Furthermore, he does not employ the notion of stimulus meaning, which was central to his doctrine of meaning and evidence in Word and Object. Similarly, he often uses the vague notion of presentations when saying what this sense of similarity operates on. Probably, Quine, who is otherwise deeply committed to clarity, uses this vague term because it allows him to avoid answering a question which is unclear to him at the time of Natural Kinds: what are the inhabitants of innate quality spaces? They cannot accommodate physical objects because this would betray his entire naturalized epistemology; neither can they accommodate patterns of stimulations, as they differ too much among humans to serve Quine's purposes.

\subsection{The Roots of Reference: Natural Selection Ensures "A Degree of Objective Validity"}

In this section, I argue that the innovation that Quine was still missing in Natural Kinds is 
introduced with the notion of perceptual similarity standards in The Roots of Reference. In this work, Quine dedicates the entire first chapter to a thorough analysis of the concepts of perception and of perceptual similarity.

Quine's introduction of the notion of perceptual similarity marks a deliberate departure from the notion of similar stimulus meaning, which dominated in Word and Object. Consider the following passage, where Quine reflects on the kinds of similarity which can be applied to the episodes of a subject's life (an episode of a subject's life is roughly the entire material constitution of an individual human at a given point in time):

Episodes in the subject's life ought, one feels, to admit of three sorts of similarity. There is receptual similarity, having to do only with sensory input. There is behavioral similarity, at the other extreme, which relates episodes according to the output of overt behavior at those times, regardless of causal factors. [...] Perceptual similarity, finally, should be somehow intermediate between receptual and behavioral similarity. It should be reflected in the behavioral output of the episode rather than just current input, but it should be reflected in only so much of the behavioral output as is somehow distinctive to the current input [emphasis RG]. (Quine 1974, 21)

According to this passage, receptual similarity is located on the level of stimulus meaning: it is a purely physiological measure of the sensory activity on a subject's nerve endings.

Obviously, we can speak of similar stimulus meanings in the sense of receptual similarity: two stimulus meanings are more or less similar to each other depending on how similar the respective global stimuli are to each other - and the similarity of global stimuli is clearly a matter of input (in the sense of impacts of particles on sensory surfaces).

Behavioral similarity operates on a different level, to wit, on the level of the reaction of the 
subject to the stimulations. Two stimulations are judged more or less similar for a subject in this behavioral sense depending on the reaction of the subject - regardless what patterns irradiate his or her nerve endings. Quine $(1974,21)$ proposes an austerely physiological measure for behavioral similarity: "the total set of fibres of striped muscle that are contracted or released on one occasion and on another".

Quine then introduces his third kind of similarity, namely perceptual similarity. Perceptual similarity is intermediate between receptual and behavioral similarity insofar as it is (1) observable in the subject's behavior and (2) relates this behavior to aspects of receptual input. As I will detail in the following paragraphs, Quine tries to deal with the first requirement by conceiving of perceptual similarity as a second-order disposition to behavior. The second requirement is fulfilled by perceptual similarity's being a disposition to acquire dispositions to act in a certain way in response to certain patterns of sensory stimulations.

Quine (1974, 8-12) conceives of dispositions as a kind of scaffold for the progress of science: by means of dispositions, we can earmark effects that we suspect have a common mechanism as their cause, without yet knowing the respective mechanism. Thus, to conceive of perceptual similarity as a kind of disposition means that we earmark a class of phenomena that we suspect are the characteristic effects of a yet unknown physical mechanism (or several such mechanisms).

The characteristic effect of perceptual similarity, conceived as a second-order disposition, is that it causes and structures first-order dispositions. To return once again to the example of the parent, the child, and the parent's uttering “Red!”, perceptual similarity enables the child to acquire the disposition to respond with "Red!" whenever it perceives a red surface. The child acquires this latter disposition because it is born with the second-order disposition to acquire first-order dispositions that closely resemble those of the parent. 
Note that Quine no more holds that similarity standards operate on global stimuli of the subject's sensory surfaces, but rather on "brief stages or temporal segments of the perceiving subject's body" (Quine 1974, 16). Obviously, this includes not only the irradiations of the subject's sensory surfaces, but also neural signals conveyed to his brain originating from his stomach, say, after a good meal. Hence, a given subject could be too sleepy to respond to a given stimulus in the way it has been conditioned to. According to Quine $(1974,22)$, this "ceteris paribus" effect is characteristic of dispositions. However, according to Quine, natural selection keeps such ceteris paribus effects under control in the case of perceptual similarity . After all, if we generally did not follow our "primitive inductions" (ibd.) acquired based on perceptual similarity (if we, for instance, do not run away from an approaching lion upon seeing it, but decide instead to give our stomach the desired rest), we would be in serious trouble.

Apart from keeping the ceteris paribus effect at bay, natural selection is also helpful in assuring that our innate sense of perceptual similarity runs "with the grain of nature":

After all, man's inductive expectations are reached by extrapolating along lines of perceptual similarity: experiences that begin similarly are expected to turn out in similar ways. Our innate standards of perceptual similarity show a gratifying tendency to run with the grain of nature. This concurrence is accountable, surely, to natural selection. Since good prediction has survival value, natural selection will have fostered perceptual similarity standards in us and in other animals that tend accordingly. (Quine 1974, 19)

After the negative assessment of our innate similarity standards in Natural Kinds - Quine wrote of the "brute irrationality of our sense of similarity" (Quine 1969b, 125) - it is surprising how respectfully Quine treats these same standards in The Roots of Reference. He 
still believes that our innate sense of similarity changes over time, but it is obvious that he has abandoned the strict distinction between a subjective, animal sense which is innate, and a much more objective, theoretical sense which is required for scientific theorizing. Furthermore, Quine holds that, since the innate standard of similarity is hereditary, "we can count on considerable social uniformity in perceptual similarity standards" (Quine 1974, 23). This means that it is rational to assume that human beings have very similar innate perceptual similarity standards.

It is remarkable how vaguely Quine expresses himself regarding the epistemic capacity of our innate perceptual similarity standards. In the passage quoted, Quine writes that they "run with the grain of nature" - without ever specifying how to read this metaphor. Similarly, immediately before this passage, Quine writes that the standards have "a degree of objective validity” (Quine 1974, 19), again without explaining this claim. Given Quine's usual clarity and explicitness, this should raise eyebrows. The explanation for this vagueness that I favor is that Quine is no scientific realist in the contemporary sense of the term; hence, he would not use a clearly realist terminology. Still, he thought that natural selection does give some epistemic validity to these evolved standards. Therefore, he uses a phrasing that gestures at such epistemic validity without committing him to anything like scientific realism (I discuss Quine's rejection of scientific realism below, section 4.1).

To conclude this discussion, it is necessary to point out that, strictly speaking, it is not perceptual similarity, but an observation sentence's similarity basis which replaces the (now untenable) notion of intersubjectively almost invariant stimulus meaning. The similarity basis of an observation sentence is "the distinctive trait shared by the episodes appropriate to that observation sentence; the shared trait in which their perceptual similarity consists" (Quine 1974, 43). Quine here explains that the similarity basis of an observation sentence is the small 
part common to all the various sensory episodes in which it is correct to utter a given observation sentence. Quine calls this part the shared trait of these episodes. Note that the similarity involved in defining similarity bases is not receptual, but perceptual. Hence, the relevant episodes can be very dissimilar on the receptual level, as long as they are perceptually similar. Accordingly, Quine uses clearly phenomenological language to illustrate his conception of trait: he writes that in the case of "Red!" the child has to learn that it is a matter of color, not of shape, of how big a patch counts as red, etc. ${ }^{11}$

Thus, unlike reception, which is "flagrantly physical", perception has "mentalistic overtones", but fortunately, it is "accessible to behavioral criteria" (Quine 1974, 4). Even though perceptual similarity still operates on physically describable events in a subject's body, there is, as it were, more to perception than meets the receptors. Quine therefore proposes a behavioral test to delineate a subject's sense of perceptual similarity:

Now we can correct our formulation of the behavioral condition for perceptual similarity, to read thus: $a$ is shown to be perceptually more similar to $b$ than to $c$ when the subject has been conditioned to respond in some fashion to all episodes in the receptual neighborhood of $b$, and to withhold that response from all those in the receptual neighborhood of $c$, and is then found to so respond to those in the neighborhood of $a$. (Quine 1974, 17-18)

According to this passage, a subject's perceptual similarity standards can be measured as follows. ${ }^{12}$ We condition the subject to respond in a certain way to a certain stimulus. Furthermore, we condition the subject not to respond thus to another stimulus. Then, we can test whether a third stimulus is perceptually more like the first than the second for the subject by stimulating the subject in the third way and observing whether the subject does or does not 
react in the manner in which the subject has been conditioned to react to the first stimulus.

Still, Quine's notion of perceptual similarity is not in the same sense physical as his notion of stimulus meaning. While, in the case of stimulus meaning, Quine could actually say what the physical structures and mechanisms consist in, perceptual similarity is rather an "earmark" (Quine 1974, 11). It refers to a class of phenomena that we suspect are the effects of a physical mechanism, or of several such physical mechanisms.

Despite this introduction and detailed discussion of perceptual similarity, Quine expresses a residual unease about the privacy of stimulus meanings in The Roots of Reference. In $\S 10$, Quine $(1974,39)$ defines observation sentences as those sentences on whose truth values, in any given situation, all competent speakers that witness the situation would agree (see also Quine 1969, 86). Hence, Quine has completely abandoned any recourse to stimulations in this definition of observation sentence. However, consider how he reflects on this definition:

The definition does raise a subtler problem, however - a problem that was already noted in another connection in $\S 6$. The definition speaks of joint witnessing. In a more precise statement, it would speak of witnesses subject to receptually similar impingements; and thus it would raise again the homology question that we noted at the end of $\S 6$.

Still, the definition is as sharp as the notion of witness and linguistic community on which it depends. It is good [sic] as behavioral concepts go. (Quine 1974, 41)

As Quine rightly points out in this passage, joint witnessing, a notion which he needs to define observation sentences, requires that witnesses can be similarly stimulated and hence provokes again the homology problem. Hence, at the end of the discussion in The Roots of Reference, Quine admits that he has not solved the basic problem caused by the fact that 
stimulus meaning is private (his gesture at the intrinsic limitations of behavioral concepts is inconclusive; Quine himself was not satisfied with this response to the issue and continued to belabor it).

\subsection{From Stimulus to Science: Natural Selection Ensures A Preestablished Harmony of Similarity Standards}

In his last monograph, From Stimulus to Science, published 1995, Quine presents an account of the infant's first steps into language where innate standards of perceptual similarity take center stage (which was not the case with Pursuit of Truth, Quine, 1992). ${ }^{13}$ Furthermore, Quine assigns evolutionary considerations a central role in his attempt to explain how communication is possible. Genuine communication requires that the recipient of a certain message can in principle understand what the sender of the message means by the message. With intersubjectively shared stimulus meanings, Quine could account for this requirement in the case of observation sentences. With private stimulus meanings, Quine is committed to the view that speakers are necessarily talking past each other even in the case of holophrastic observation sentences, as no two speakers could possibly mean the same thing with any given observation sentence (remember that Quine intended stimulus meaning as a replacement for traditional mentalistic meaning).

Because standards of perceptual similarity are required for first-language acquisition, indeed, for all learning, we are born with a provisional standard of perceptual similarity. After enlisting natural selection for a first time to ensure that our innate standards "mesh pretty well with natural trends”, Quine $(1995,20)$ rephrases the puzzle of shared circumstances and sketches the requirements for a solution: ${ }^{14}$ 
There is a puzzle here. Global stimuli are private: each is a temporally ordered set of some one individual's receptors. Their perceptual similarity, in part innate and in part molded by experience, is private as well. Whence then this coordination of behavior across the tribe? It requires that if two individuals jointly witness one scene, and subsequently jointly witness another scene, and the one witness's global stimulations on the two occasions qualify for him as perceptually similar, usually the other witness's stimulations will also so qualify for the other witness. (Quine 1995, 20)

In this passage, Quine first reaffirms the presuppositions of the puzzle. In contrast to the account given in Word and Object, global stimuli are private, and so is perceptual similarity. The problem emerges if these private entities should function as the building blocks of something decidedly public, namely of language. If we experience situations differently, and if we find different experiences similar, it seems impossible to explain how we have managed to develop a common language, and it seems deeply mysterious that we should even agree on what to say regarding a specific situation. Furthermore, Quine gives a very succinct wording of what is required to dispense with this mystery. If two speakers of a language witness two scenes, they must unanimously perceive the respective global stimuli as similar or not.

In accordance with his overall naturalized epistemology, Quine makes global stimuli, and not situations, the objects of comparisons. The requirement, however, irreducibly involves these shared situations: the two speakers must agree on their verdict regarding global stimuli of two situations or scenes. Thereby, the main challenge is that these two situations give rise to four different global stimuli - one global stimulus for each speaker in each situation. Therefore, in each of the two situations, speaker $A$ must consider two global stimuli as similar (or dissimilar) which differ from the stimuli that speaker $B$ must judge as similar (or dissimilar). The challenge therefore is not simply to explain why speakers find the same situations 
perceptually similar, but rather why they find the incongruent respective global stimuli that arise from these situations perceptually similar. Strictly speaking, the scenes for the two speakers are never the same, since they always experience different global stimuli.

A first step to solving this puzzle has already been taken in The Roots of Reference: Quine proposes to focus on perception, as opposed to reception (compare above, p. 14). Quine's conception of perception allows for perceptual similarity in spite of receptual dissimilarity. There is no principled reason why two subjects should not find receptually dissimilar situations perceptually similar. The crucial question therefore is: why should these innate perceptual similarity standards be so constituted that the speakers will, in spite of receptually dissimilar global stimuli, perceive the same situations as similar? Quine adresses this question in the following passage:

So we see a preestablished harmony of perceptual similarity standards. If two scenes trigger perceptually similar global stimuli in one witness, they are apt to do likewise in another.

This public harmony of private standards of perceptual similarity is accounted for by natural selection. The individual's initial standards of perceptual similarity are inculcated, we saw, by natural selection, and so, thanks to shared ancestry and shared environment, will tend to harmonize across the tribe. The changes in standards subsequent to birth will also tend to harmonize, because of the shared society and environment. (Quine 1995, 21)

According to this passage, there is a preestablished harmony of perceptual similarity standards among human beings. ${ }^{15}$ This preestablished harmony ensures that, if two scenes cause perceptually similar global stimuli in one human, they typically also cause perceptually 
similar global stimuli in any other human.

Furthermore, Quine claims that this preestablished harmony can be explained by natural selection, as far as the innate perceptual similarity standards are concerned. These standards stand in a preestablished harmony with our fellow human beings' because they are genetic traits, which are inherited from our common ancestors, and which have developed in a shared environment.

Finally, these parts of the standards that are not innate are uniform among a speech community because of "shared society and environment". This means that they are uniform because we live in the same society and in the same environment.

Quine cherishes the preestablished harmony of innate perceptual similarity standards because he thinks it allows him to explain how real communication is possible despite the privacy of stimulus meanings and without presupposing any pre-linguistic perception as of physical objects. By 'real' or 'genuine' communication, I mean communication where the meanings of some basic linguistic structures, namely holophrastic observation sentences, are shared among speakers. ${ }^{16}$

According to this account, first-language acquisition works in spite, and not because of the patterns of sensory stimulations that affect both the infant and the parent. As shown above, for Quine, the infant and the parent do not witness the same situation; rather, they are experiencing different global stimuli in the same situation (the sameness of the situation being irrelevant for Quine's accounts of meaning and evidence). This means that the Quinean subject, while not being causally entirely windowless, like the Leibnizian monad, is still experiencing sensory stimulation as something that separates her from, rather than connects her to, her fellow humans. ${ }^{17}$ 
This implies nothing less than that Quine has decisively rejected core elements of his empiricism as well as his physicalism: the empirical data, which Quine conceives as physical sensory stimulations, are not something that connect us to the world and to our fellow humans, but rather as something that separates us from both and that has to be overcome with innate, biologically understood cognitive capacities. This amounts to a rejection of core elements of Quine's empiricism because these cognitive capacities, and not empirical data of some sort, are now the crucial element of Quine's explanation of communication and of his theory of empirical evidence. It amounts to a rejection of physicalism because Quine's explanation is squarely based in the biological realm, with no indication how it could be (ontologically or semantically) reduced to physics. ${ }^{18}$

\section{Critical Assessment of Quine's Mature Theory of Meaning and}

\section{Evidence}

In this section, I critically assess Quine's mature view that natural selection furnishes a degree of objective validity to the similarity standards of humans; then, I question Quine's use of natural selection to explain the very possibility of genuine communication.

\subsection{Can Natural Selection Ensure "A Degree of Objective}

\section{Validity"?}

We have seen that, in his Natural Kinds, Quine toyed with the idea that cognitive capacities that are the product of natural selection are irrational and useless for science. Later on, however, he credited these same products with the ability to achieve a degree of objective validity. We still find these two positions in contemporary debates. The view of naturally selected abilities as irrational is basically the line of reasoning developed by Alvin Plantinga 
in The Evolutionary Argument Against Naturalism. In short, Plantinga (2002) argues that if our cognitive faculties are the product of evolution by natural selection, it is probable that naturalism is false, as evolution does not select for truth-conducive abilities, but only for abilities which foster survival. Since the latter are distinct from the former, Darwinists must conclude that, while their Darwinism may help them survive, it has nothing to do with truth. In contrast, Hilary Kornblith explicitly argues for a continuity between the kind of reasoning that fosters survival and the kind of reasoning that is at work in successful scientific theories (Kornblith 1993, 61ff., and Kornblith 2014, 132). Furthermore, Kornblith $(2014,126)$ makes clear that he commits himself to the claim that "natural selection is selecting for knowledgeacquiring capacities - that is, processes of belief acquisition that tend to produce truths". In contrast to both positions, Maddy $(2017,219)$ and Burge $(2010,308)$ basically hold that some mental ability's being the product of natural selection has no implications regarding the ability's capacity to, as it were, deliver truths about the world.

For present purposes, however, I think it more important to point out that whether we buy into Quine's claim regarding the degree of objective validity depends centrally on how such objective validity is conceived. If it is understood in the sense of contemporary scientific realism, as correctly representing mind- and theory-independent reality, ${ }^{19}$ then Quine does face the debate sketched above.

However, I think it misguided to read Quine as a contemporary scientific realist; instead, it seems more convincing to conceive his 'robust realism' as a position sui generis. To see why, consider the following passage:

But I also expressed, at the beginning, my unswerving belief in external things people, nerve endings, sticks, stones. This I reaffirm. I believe also, if less firmly, in atoms and electrons and in classes. Now how is all this robust realism to be reconciled 
with the barren scene that I have just been depicting? The answer is naturalism: the recognition that it is within science itself, and not in some prior philosophy, that reality is to be identified and described.

The semantical considerations that seemed to undermine all this were concerned not with assessing reality but with analyzing method and evidence. They belong not to ontology but to the methodology of ontology, and thus to epistemology. Those considerations showed that I could indeed turn my back on my external things and classes and ride the proxy functions to something strange and different without doing violence to any evidence [emphasis RG]. But all ascription of reality must come rather from within one's theory of the world; it is incoherent otherwise. (Quine 1981, 21)

In this important passage, Quine first specifies what he means by 'robust realism'. Quine writes that he unswervingly believes in external things, notwithstanding his insistence that all these external things are theoretical posits that we could replace by "something strange and different" through a proxy function. It is essential to Quine's robust realism that it incorporates this insight. Such a position is inconsistent with contemporary scientific realism. Scientific realists are precisely contesting that kinds such as atoms or electrons are real only in the sense that they are posited by a predictively highly successful theory: they insist that they are part of the furniture of the world, conceived mind- and theory-independently, in addition to being part of our theories. Quine's robust realism is restricted to the first assertion. This reading of Quine's realism is congenial to Ben-Menahem (2016), who urges that Quine, like James, rejected the very possibility of comparing our categories with the world's very own joints.

Furthermore, Quine emphasizes that this combination of belief in external things with belief that our evidence does not determine that there are material objects rather than quadruples of 
numbers is underwritten by his naturalism. This naturalism is based on the insight that reality must be described from within science, and not in some 'prior philosophy', a term that Quine uses interchangeably with 'first philosophy'. Hence, for Quine, it is a consequence of the rejection of first philosophy that realism cannot amount to more than the robust or immanent realism that he has just introduced: unless we have a kind of access to reality that is qualitatively different from the one provided by empirical science, we cannot discern whether the posits of our most successful theories are real in a sense other than being such posits. Epistemically speaking, there is no other way to justify a posit than the empirical-scientific way. If this way leads to the insight that other posits would be just as justified, as Quine thinks is the case, we have to acknowledge this, but we are not committed to reject the posits that were used to arrive at this insight.

Caruso (2007) also examines the relationship between Quine's robust realism and scientific realism and reaches a position that is congenial to mine. Caruso $(2007,80)$ maintains that Quine's realism requires “taking one's conceptual scheme seriously and owning the beliefs of the moment", while insisting that the reality posited by the scheme is "constituted by our imposition of concepts, theories, and language". Furthermore, this reading of Quine's realism agrees with Keskinen $(2012,144)$ in its rejection of conceiving Quine as a scientific realist: "with regard to the reality of objects in some further sense than being posits of our science, Quine's epistemology does not entail anything $[\ldots]$ ”.

This implies that Quine's assertion that our innate sense of similarity has "a degree of objective validity" should not be read as: correctly prefigures mind-and theory-independent reality. Rather, it expresses the claim that this sense has some epistemic validity and hence some rationality - if only of an instrumental sort. Still, it strongly contrasts with the view taken in Natural Kinds, which asserted the brute irrationality of this sense. 
It is no chance that the one article where Quine comes closest to embrace a fully-fledged scientific realism is also the one article where he proposes that we have to transcend our innate sense of similarity to properly engage in science. The higher your conception of the epistemic power of science, the more difficult it will be for you to explain humans' ability to pursue such science with recourse to natural selection.

However, by means of conclusion, I submit that Quine's position on the issue is largely consistent and convincing. Across his publications from Word and Object onwards, Quine has, with very few exceptions such as Natural Kinds, embraced an instrumental conception of scientific success: science is successful insofar as it helps us predict future phenomena, which is of obvious survival value, and hence increases humans' reproductive fitness. This suggests that if Quine claims that our innate sense of similarity, and hence the science that is based on it, has a degree of objective validity, this means little more than that it is a good, and hence instrumentally rational means to cognitively cope with our environment; and to say that natural selection favors capacities that help to cope with the environment is almost tautologous.

\subsection{Can Natural Selection Guarantee the Preestablished Harmony?}

The second use to which Quine puts natural selection is to ensure that there exists a preestablished harmony of innate perceptual similarity standards such that two humans typically perceive the same situations similarly despite their being stimulated very differently. At first sight, this appears to be an analogous claim to the one examined above regarding objective validity: It seems clear that it is of survival value if two people can successfully communicate about events in their environment. 
However, the situation here is substantially more complex. Rather than merely reaffirming that natural selection selects for capacities that help us to successfully cope with our environment, Quine here enlists natural selection to solve a very specific problem in his elaborate account of communication. Regarding the solution to this problem, it is crucial to distinguish between some feature's being plausibly the result of a process of random variation and natural selection (because the feature is prima facie conducive to fitness) and there being a fully-fledged, evolutionary-biological account of how that same feature has resulted from this process.

It would certainly be unfair to ask Quine for such a fully-fledged account of the evolution of the preestablished harmony of perceptual similarity standards. After all, Quine $(1974,3)$ explicitly maintains that his project "may be pursued at one or more removes from the laboratory, one or another level of speculativity." In this vein, he could respond that his invocations of natural selection do not have to satisfy fully-fledged scientific standards, since he operates on the more speculative level, where he sketches a new research perspective that may then be fleshed out by trained specialists.

Hence, the interesting question is: is it plausible that it is possible for biologists to provide such a fully-fledged evolutionary-scientific account of the phenomenon in question? The phenomenon in question is the preestablished harmony of innate perceptual similarity standards, which is the cornerstone of Quine's account how genuine communication is possible at least on the level of holophrastic observation sentences.

In the remainder of this section, I raise doubts regarding this possibility. My entire discussion is heavily indebted to Richardson (2007), who insists that some subject matters are wellsuited for evolutionary studies, while others are not:

Fruit flies are a well-adapted subject for evolutionary studies. Likewise, as I 
illustrated in the previous chapter, the morphology of Gammarus minus is one that is amenable to evolutionary analysis. But psychology, in contrast, is poorly adapted as an evolutionary science. (Richardson 2007, 148)

Richardson here essentially claims that the methods that proved successful in the case of fruit flies and in the case of the morphology of Gammarus minus (a species of amphipod crustacean) are likely to be not equally successful when applied to psychology. The reason for this is that it is much more difficult to examine human psychology in a way that satisfies core methodological principles of evolutionary biology than it is to examine the morphology of Gammarus minus in such a way. As will become evident, this has much to do with the amount of evidence and information that is available about the morphology of Gammarus minus, but not with regard to human psychology.

In the following, I introduce these elements, or pieces of information, that an ideal explanation in evolutionary biology requires, following Richardson (2007, 99-104; he himself refers to Brandon 1990, chap. 5). Richardson admits that an explanation by natural selection does not have to detail all of these pieces to pass muster; but they do give an idea of the "sort of criteria relevant to deciding whether an explanation passes muster" (Richardson 2007, 99). There are five such elements.

Selection. First, Richardson requires that "There must be evidence that selection has in fact occurred". This in turn requires that we have information regarding the variation of the feature in question by the time the natural selection is postulated to have occurred, together with information about the differences in terms of survival and reproductive success that these different features have bestowed upon their bearers.

Ecological Factors. Next, Richardson requires that we can explain said selection with recourse to ecological factors, that is, we need to be able to specify which properties of the 
social or physical environment caused a certain trait to be conducive to its bearer's reproductive and survival success.

Heritability. For evolution through natural selection to be possible, the features that increase the reproductive fitness of their bearers must be heritable. This means, according to Richardson (ibid.), that phenotypical features should be correlated between parent and offspring to an extent that is clearly greater than mere chance. This requires that if the parent has a specific selective advantage, it should be more likely than not that the parent passes this advantage on to the offspring.

Population Structure. The gist of this factor is that if there are different populations living in different habitats that furnish different biological features with a selective advantage, then a gene flow between these populations is likely to cancel out any selection due to the several different habitats. For instance, assume that there are two populations of fruit flies, one living by a pond, the other living in a swamp. The populations are sufficiently close to allow for frequent mating between members of the two populations. Furthermore, assume that large fruit flies living in the swamp have more offspring than small ones living in the swamp, while the converse holds for fruit flies living by the pool. In such a situation, the two selective pressures are likely to cancel each other out: there will be no significant increase or decrease in size of the fruit flies in either population across generations because of inter-population mating.

In contrast, a completely isolated population in a uniform environment that exercises a consistent selective pressure on the population is more hospitable to evolution through natural selection. For instance, assume that there is just the one population living in the swamp, where larger flies have significantly more offspring. Then, there may occur a selection and an increase in size of individual flies across generations. 
Trait Polarity. Richardson uses this condition to emphasize that there is a difference between a feature's being adaptive and a feature's being an adaptation. In particular, there can be adaptive features that are not adaptations. This is the case if a certain biological feature that is very useful, perhaps even indispensable for the life-form of a given species, is present in ancestral species already. Richardson (ibid.) mentions the skull sutures of mammal infants. Without them, mammalian birth would probably be impossible, so this feature is very adaptive. However, since it is present in birds and reptiles, it cannot possibly be an adaptation to said mammalian birth: the skull sutures existed well before mammalian birth evolved.

To illustrate how these elements are included in state-of-the-art explanations by natural selection, Richardson $(2007,120-24)$ presents the case of Gammarus minus. This species of amphipod crustacean has specific adaptations for its habitat, dark caves in West Virginia. Individuals belonging to this species differ from their non-cave-inhabiting ancestors mainly in being larger, having drastically reduced eyes, and having larger antennae. The question to be answered in the following is whether these differences can be explained with recourse to natural selection. Regarding selection, it is easy to verify that there is variation of the trait: one can simply measure body and antenna size and count the eye facets of individuals living in the caves. Then, one can compare these numbers to the ones of their relatives that do not live in caves but rather near springs.

Furthermore, in the habitat of Gammarus minus, which is completely dark, larger antenna are obviously conducive to survival. More precisely, concerning the ecological factors that drive the selection, Richardson $(2007,122-23)$ mentions that field studies in caves show that “individuals with smaller eyes, larger antennae, and larger body size mate more frequently and produce more offspring than those in the same population with larger eyes and smaller antennae and body size.” Regarding heritability, Richardson $(2007,123)$ introduces a 
laboratory study that indicates that offspring of cave-dwelling Gammarus have smaller eyes than offspring of spring-dwelling Gammarus, with a correspondence that roughly matches the heritability of body height in humans. Concerning population structure, it has been verified that the different populations of Gammarus minus are very isolated and exposed to a very homogeneously light-less environment (Richardson 2007, 123-24). Trait polarity, finally, is very easily established, as the non-cave-dwelling Gammarus have substantially bigger eyes, but shorter bodies and antenna.

Now, let us consider Quine's explanation by natural selection of the preestablished harmony of humans' innate perceptual similarity standards. Of the five pieces of information that Richardson requires from an ideal explanation by natural selection, Quine only touches upon two: selection and ecological factors. Concerning selection, he does not specify the variation of similarity standards between individuals, nor does he specify the time where the selection is supposed to have occurred. In terms of the ecological factors that have conferred a survival advantage on their bearers, Quine does not get more specific than "the grain of nature" or "natural trends". There is no information on heritability, population structure, or on trait polarity. In short, it is obvious that Quine's invocations of natural selection fall short of the standards employed by the science of evolutionary biology.

However, as mentioned above, the interesting question is not whether Quine's own invocations fulfill these standards, but rather whether it is possible that evolutionary biologists develop an account along Quine's lines that fulfills these standards. There are good reasons to assume that this is not possible. Consider the five kinds of information that Richardson lists for an ideal explanation of a human trait by natural selection. Take as the trait in question the preestablished harmony of human perceptual similarity standards, which explains the possibility of communication. Skulls and bones cannot give us any information 
regarding the variation of innate perceptual similarity standards, since they are behavioral not anatomical traits. Similarly, we have no clue as to the ecological factors that shaped these standards, or about the relevant population structure and the trait polarity. More importantly, it seems very unlikely that we will ever have such information available.

Hence, while it is not impossible that, at some point, we will have all the information necessary for a proper evolutionary explanation to cash out Quine's speculations, or, alternatively, that the methodological standards for such an explanation (perhaps due to vastly more reliable computer simulations) change drastically, it is doubtful whether there will be such an explanation. This means that it is doubtful whether Quine is able to explain why there is such a thing as successful communication, even on the level of holophrastic observation sentences.

\section{Conclusion}

In this article, I have delineated the reasons that prompted Quine to abandon core elements of his empiricism and physicalism: the discovery that nerve endings are non-homologous lead Quine to abandon the notion of intersubjectively (almost) invariant stimulus meaning in favor, eventually, of a preestablished harmony of innate perceptual similarity standards. This preestablished harmony is accounted for by natural selection, and it ensures that two humans understand each other on the level of holophrastic observation sentences despite the incongruent empirical data that they receive in any given situation.

Quine also enlists natural selection to explain the success of science. While, in Natural Kinds, Quine has considered the innate sense of perceptual similarity an obstacle to theoretical science, his mature position is that the success of science can be explained by appreciating that the similarity standards on which this science is ultimately based have been selected for 
precisely such a success.

In a critical assessment of these uses of natural selection, I have suggested that Quine is justified in the first use, whereas it is doubtful whether he succeeds in using natural selection to guarantee the preestablished harmony of innate perceptual similarity standards. This implies that it is unclear whether Quine can explain how genuine communication is so much as possible.

\section{References}

Ben-Menahem, Yemima. 2016. "The Web and the Tree: Quine and James on the Growth of Knowledge." In Quine and His Place in History, ed. Frederique Janssen-Lauret and Gary Kemp, 59-75. Palgrave Macmillan.

van Brakel, Jaap. 2000. “Quine and Innate Similarity Spaces.” In Quine. Naturalized Epistemology, Perceptual Knowledge and Ontology, ed. Lieven Decock and Leon Horsten, 70:81-99. Poznan Studies in the Philosophy of the Sciences and the Humanities. Rodopi. Brandon, Robert N. 1990. Adaptation and Environment. Princeton University Press.

Broughton, Lynne M. 1981. “Quine’s Quality Space.” Dialectica 35:291-302.

Burge, Tyler. 2010. Origins of Objectivity. Oxford University Press.

Caruso, Gregg. 2007. "Realism, Naturalism, and Pragmatism: A Closer Look at the Views of Quine and Devitt." Kriterion - Journal of Philosophy 21:64-83.

Chakravartty, Anjan. 2017. "Scientific Realism.” In The Stanford Encyclopedia of Philosophy, ed. Edward N. Zalta. Stanford University. https://plato.stanford.edu/archives/sum2017/entries/scientific-realism/.

Devitt, Michael. 1997. Realism and Truth. Princeton University Press. 
Ebbs, Gary. 2016. 'Introduction to 'Preestablished Harmony' and 'Response to Gary Ebbs'."' In Quine and His Place in History, ed. Frederique Janssen-Lauret and Gary Kemp, 21-28. Palgrave Macmillan.

Føllesdal, Dagfinn. 2014. “Developments in Quine’s Behaviorism.” In A Companion to W.V.O. Quine, ed. Gilbert Harman and Ernie Lepore, 263-78. John Wiley \& Sons.

Gibson, Roger F. 1988. Enlightened Empiricism. An Examination of W. V. Quine's Theory of Knowledge. University of South Florida Press.

Gjelsvik, Olav. 2014. “Quine on Observationality.” In A Companion to W.V.O. Quine, ed. Gilbert Harman and Ernie Lepore, 313-32. John Wiley \& Sons.

Glock, Hans-Johann. 2003. Quine and Davidson on Language, Thought and Reality. Cambridge University Press.

Hylton, Peter. 2007. Quine. Routledge.

Janssen-Lauret, Frederique. 2016. "Meta-Ontology, Naturalism, and the Quine-Barcan Marcus Debate." In Quine and His Place in History, ed. Frederique Janssen-Lauret and Gary Kemp, 147-167. Palgrave Macmillan.

Johnsen, Bredo C. 2014. “Reclaiming Quine’s Epistemology.” Synthese 191:961-88.

Keskinen, Antti. 2012. “Quine on Objects: Realism or Anti-Realism?” Theoria 78:128-45.

Kornblith, Hilary. 1993. Inductive Inference and Its Natural Ground. The MIT Press.

- 2002. Knowledge and Its Place in Nature. Oxford University Press.

—. 2014. "Knowledge in Humans and Other Animals." In Selected Essays, 119-41.

Oxford University Press.

Kuhn, Thomas S. 1962/1996. The Structure of Scientific Revolutions. University of Chicago 
Press.

Maddy, Penelope. 2017. What Do Philosophers Do? Skepticism and the Practice of Philosophy. The Romanell Lectures. Oxford University Press.

Plantinga, Alvin. 2002. “The Evolutionary Argument Against Naturalism.” In Naturalism Defeated? Essays on Plantinga's Evolutionary Argument Against Naturalism, ed. James Beilby, 1-12. Cornell University Press.

Psillos, Stathis. 2009. Knowing the Structure of Nature. Palgrave MacMillan.

Quine, Willard Van Orman. 1960. Word and Object. The MIT Press.

—. 1951/1980. “Two Dogmas of Empiricism”. In: From a Logical Point of View, 20-46. Harvard University Press.

_. 1969a. "Epistemology Naturalized." In Ontological Relativity and Other Essays, 6990. Columbia University Press.

—. 1969b. "Natural Kinds.” In Ontological Relativity and Other Essays, 114-38.

Columbia University Press.

1969c. "Propositional Objects.” In Ontological Relativity and Other Essays, 139-60.

Columbia University Press.

- 1974. The Roots of Reference. Open Court Publishing Co.

_. 1981. "Things and Their Place in Theories". In: Theories and Things. Harvard

University Press, 1-23.

- 1992. Pursuit of Truth. 2nd ed. Harvard University Press.

— 1995. From Stimulus to Science. Harvard University Press.

—. 1996. "Progress on Two Fronts.” In Quintessence, ed. Roger F. Gibson, 169-173. 
Harvard University Press.

_. 1995/2016a. "Response to Gary Ebbs." In Quine and His Place in History, ed. Frederique Janssen-Lauret and Gary Kemp, 33-36. Palgrave Macmillan.

1995/2016b. "Preestablished Harmony" In Quine and His Place in History, ed.

Frederique Janssen-Lauret and Gary Kemp, 29-32. Palgrave Macmillan.

Richardson, Robert C. 2007. Evolutionary Psychology as Maladapted Psychology. The MIT Press.

\section{Footnotes}

1 Note that I am not claiming any close connections between physicalism as such and empiricism as such; one can defend the one without defending the other.

2 I am here not referring to the indeterminacy of translation. The essential point here is that Quine cannot explain communication at a very basic level, a level that Quine did not want to be touched by any indeterminacy, namely the level of holophrastic observation sentences. As I detail on pages 4-5, Quine intended holophrastic observation sentences to furnish true intersubjectivity, almost entirely unaffected by indeterminacies.

3 This leaves room for forms of communication (which one might or might not call languages) that lack any such grammatical or ontological structure. Observation sentences of these forms of communications could then only be used holophrastically. See Janssen-Lauret $(2016,149)$.

${ }^{4}$ Thus Quine's use of the linguistic term 'holophrastic', which normally refers to the one- 
word sentences that a child produces at an early stage of language learning.

${ }^{5}$ Quine gives the final definition of stimulus meaning as follows: "a stimulus meaning is the stimulus meaning modulo $n$ seconds of sentence S for speaker $a$ at time $t$ " (Quine 1960, 33). The specification of the modulus and the time are required to avoid some technical problems. For my purposes, however, it is more expedient to focus on the first definition, as it is more straightforward.

${ }^{6}$ Føllesdal $(2014,268)$ worries that taking sensory stimulations as basic semantic units of significance contradicts Quine's behaviorism, since “they are not part of the publicly available evidence that is so crucial to behaviorism". I think this worry can be met in the following way: just take the subject into a laboratory, where behaviorism is naturally at home, and wire it up such that the researchers can monitor all the activities on the subject's nerve endings. This furnishes all the public availability that Quine's behaviorism requires. This largely agrees with Johnsen $(2014,984)$, who argues that the stimulus meaning is not what any competent speaker means with a sentence, but what external researchers are able to determine as such.

${ }^{7}$ There is surprisingly little research on Quine's notion of quality space. Exceptions are Broughton (1981), who suspects that Quine's notion is covertly mentalistic, and van Brakel (2000), who compares Quine's notion to similar conceptions in cognitive science.

${ }^{8}$ Note that the intentional vocabulary is here merely to facilitate presentation. The account to which Quine ultimately commits himself is phrased in terms of efficient causes, dispositions, stimuli, and conditioned responses.

${ }^{9}$ In this context, 'homology' means having the same, or at least a very similar structure. Hence, the nerve endings of two humans are homologous if they have the same structure. This requires that their sensory surfaces are isomorphic. 
This passage resounds in Pursuit of Truth, where Quine claims that he felt discomfort over the notion of sameness of stimulus meanings already in 1965, and he expresses his conviction that "anatomical minutiae" like homology of nerve endings should be irrelevant to his project, see Quine $(1992,40)$ and Quine $(1992,3-4)$.

${ }^{10}$ Quine's use of phrases such as the cosmic machine revealed by science (Quine 1969b, 133) is certainly notable. I discuss below (section 4.1), why the use of these metaphors does not imply that Quine defends anything close to contemporary scientific realism. Still, these phrases explain why naturalistic realists like Kornblith (2002) are particularly fond of Natural Kinds.

${ }^{11}$ This has led Føllesdal $(2014,276)$ to claim that Quine's position is surprisingly similar to Husserl's.

${ }^{12}$ The notion of a receptual neighborhood is intended to allow for perceptual similarity to operate on patterns of stimulations that are receptually similar, but not identical.

${ }^{13}$ In his "Progress on Two Fronts" (Quine 1996), Quine gives an account of the preestablished harmony of innate perceptual similarity standards, and of its significance for his theory of meaning and evidence, that is very similar to the one in From Stimulus to Science.

${ }^{14}$ The vague expression "mesh pretty well with natural trends" is another Quinean attempt to say that evolution ensures that these standards have a certain epistemic validity, without committing himself to anything like scientific realism. Compare below, section 4.1.

${ }^{15}$ See also the very similar passages on Quine (1995/2016a, 31) as well as Quine (1995/2016b, 35). The passages show how central to Quine's thinking preestablished harmony was in this late stage of his publishing career.

${ }^{16}$ Compare the following passage from a letter by Quine to Gary Ebbs (Ebbs 2016, 28): "The one major departure from Word and Object in my later work, so far as I see, is renunciation 
of intersubjective identity or similarity of stimulus meanings. Preestablished harmony of subjective standards of subjective perceptuality fills the bill."

${ }^{17}$ Compare Gjelsvik (2014, 322), who also sees this change as a radical modification of Quine's conception of evidence.

${ }^{18}$ See also Quine (1995/2016a, 29ff.), where Quine emphasizes publicly observable behavior at the cost of intersubjectively shared stimuli - and then gives an account of how preestablished harmony of innate perceptual similarity standards is needed to explain firstlanguage acquisition based on such publicly observable behavior. This nicely agrees with my position, according to which the preestablished harmony is needed to, as it were, compensate for the privacy of stimulus meanings. I take the following paragraph from said article (Quine 1995/2016a, 30f.) to express exactly this position:

In Pursuit of Truth that appeal [to intersubjective similarity of stimulus meanings, $R G$ ] is out of order, so the causal question recurs: why, after the mother has got the child to affirm 'Milk' once in an appropriate situation, does the child's usage continue to agree with the mother's? The answer can lie no longer in intersubjective similarity of stimulus meaning. It now lies rather in an intersubjective parallelism of subjective scales of perceptual similarity [emphasis RG]. If $A$ and $B$ jointly witness two events, and $A$ 's neural intakes on the two occasions are perceptually similar by $A$ 's standards, then $B^{\prime}$ s intakes will tend to be similar by $B$ 's.

${ }^{19}$ Compare Devitt (1997, 14-22), Psillos (2009, 34), and Chakravartty (2017). 